\title{
Proposition de nouvelles approches dans l'étude des hameçons océaniens
}

\section{Christelle Carlier et Éric Conte}

\section{Q OpenEdition \\ 1 Journals}

Édition électronique

URL : http://journals.openedition.org/jso/5742

DOI : $10.4000 /$ jso. 5742

ISSN : $1760-7256$

Éditeur

Société des océanistes

\section{Édition imprimée}

Date de publication : 30 juin 2009

Pagination : 133-146

ISBN : 978-2-85430-024-6

ISSN : 0300-953x

Référence électronique

Christelle Carlier et Éric Conte, " Proposition de nouvelles approches dans l'étude des hameçons océaniens ", Journal de la Société des Océanistes [En ligne], 128 | janvier-juin 2009, mis en ligne le 30 juin 2012, consulté le 01 mai 2019. URL : http://journals.openedition.org/jso/5742 ; DOI : 10.4000/ jso. 5742

\section{(ㄷ) Tous droits réservés}




\section{Proposition de nouvelles approches dans l'étude des hameçons océaniens}

par

Christelle CARLIER* et Éric CONTE**

\section{RÉSUMÉ}

Dans le prolongement des travaux de José Garanger sur les hameçons océaniens, il est proposé une méthode objective de prise de mesures sur le matériel et, sur une collection provenant du site de Manihina (Ua Huka, archipel des Marquises), est développé un moyen de reconstituer, à partir des nombreux fragments retrouvés en fouille, la forme originelle et la taille des hameçons simples.

Mots-CLÉs: hameçon, méthodologie, Polynésie, Manihina, Ua Huka, îles Marquises

Depuis le début des fouilles archéologiques en Polynésie orientale, faute de poterie, on a été tenté d'utiliser les hameçons comme indicateurs chrono-culturels. Une démarche dont la pertinence mériterait d'être examinée de façon critique en regard, notamment, des nouvelles datations obtenues ces dernières années sur les principaux sites d'où provenaient les hameçons concernés. Ce faisant, on a quelque peu oublié l'essentiel: les hameçons sont un élément indispensable pour reconstituer les techniques de pêche, un aspect déterminant de la vie des anciens Polynésiens. Cette dimension fonctionnelle n'est toutefois accessible que si l'on est en mesure d'établir une relation entre un type et un module d'hameçon, une stratégie de

\begin{abstract}
Extending José Garanger's work on Oceanian fishhooks, this paper proposes a new objective method of measurements. To study the fish hook fragments found in large quantities in excavations, a process of morphological reconstruction has been developed and tested on a collection from the Manihina site (Ua Huka, Marquesas islands). This method can be used to identify the original shape and size of simple fishooks.
\end{abstract}

KEYwORDS: Fishhook, methodology, Polynesia, Manihina, Ua Huka, Marquesas islands

pêche et une proie visée, ce que contribuent à documenter les démarches ethno-archéologiques parfaitement justifiées par le contexte écologique, historique et culturel polynésien (Conte, 2000). De plus, à travers les chaînes opératoires, les procédés utilisés pour la réalisation des hameçons, peut être entrevu le savoirfaire technique, voire la dimension cognitive de la relation de ces hommes aux matériaux employés. Quel que soit le but recherché, pour pouvoir travailler sur les hameçons retrouvés en fouille, il importe, comme pour tous les artefacts, d'opérer des classifications typologiques, d'effectuer des mesures et de faire des constats quant aux procédés de fabrication utilisés pour les produire.

* Doctorante, Université Paris-I, umR 7041, christelle_carlier@hotmail.fr.

** Professeur, Université de la Polynésie française, uMR 7041, eric.conte@upf.pf. 
C'est à cette exigence que répondait José Garanger dans son fameux article de 1965, «Hameçons océaniens : éléments de typologie », qui offrait, comme il le fera plus tard pour les herminettes, des éléments de description systématiques et rationnels, des indications précises sur les mesures pertinentes. Cette contribution devait être d'un grand secours pour tous ceux qui, après lui, eurent à étudier ce type de matériel et elle ouvrait la possibilité d'élaborer une base de données sur les hameçons océaniens qui, hélas, fait encore défaut. Soucieux de clarté et de pédagogie, José Garanger y faisait également l'effort d'adjoindre à son article un lexique anglais-français des principaux termes descriptifs des hameçons, rendant plus intelligibles les travaux des chercheurs anglo-saxons qui s'étaient déjà intéressés à ces questions, notamment K. Emory, W. Bonk et Y. H. Sinoto (1959) et R. C. Suggs (1961). Depuis cette publication, nombreuses ont été les études consacrées à l'analyse des hameçons. Citons parmi d'autres: J. Davidson (1971, 1976, 1996) à Nukuoro, B. V. Rolett (1998) à Tahuata, P. Ottino (1992-a, 1992-b) à Ua Pou et M. S. Allen (1996) à Aitutaki.

Toutefois, l'examen de ces travaux montre qu'une certaine part de subjectivité peut intervenir, notamment dans la prise des mesures, aboutissant parfois à des résultats contestables. De plus, les études portent pour l'essentiel sur les hameçons complets alors qu'ils sont en général très minoritaires dans les corpus, tandis que les fragments, plus nombreux, ne sont guère exploités.

C'est à ces deux problèmes que cet article se propose d'apporter des éléments de réponse. Sur les mesures, après avoir montré les approximations auxquelles conduisent dans certains cas les prises de mesures habituelles, nous présenterons une nouvelle méthode se voulant la plus systématique et objective possible. En deuxième lieu, nous exposerons un procédé de reconstitution des hameçons entiers à partir des fragments que nous appliquerons, afin d'en tester l'efficacité, à une collection provenant du site de Manihina, sur l'île Ua Huka (archipel des Marquises).

\section{Les prises de mesures des hameçons simples}

En préambule, rappelons la terminologie définie par José Garanger (1965 : 131) selon laquelle les parties constitutives d'un hameçon simple, c'est-à-dire fait d'une seule pièce, sont les suivantes (figure 1):

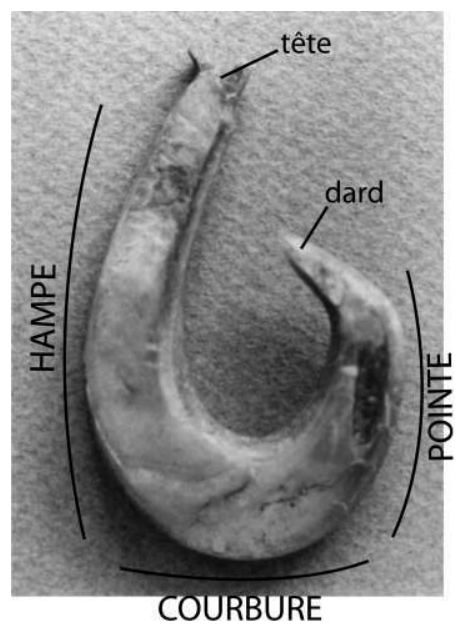

FIGURE 1. - Les différentes parties composant un hameçon simple

- La hampe est surmontée par la tête de hampe servant à la fixation de la ligne.

- La tête est un élément morphologique important car sa forme donne non seulement des informations technologiques liées à l'attache de la ligne, mais a également été considérée (Sinoto, 1975:1) comme pouvant servir d'indicateur chronologique.

- La courbure relie le bas de la hampe et la pointe. Elle doit être la partie la plus solide de l'hameçon car c'est à son niveau que se concentrent les tensions exercées lors de la capture.

- La pointe est droite ou incurvée. Elle peut être munie d'un ardillon intérieur ou extérieur, voire de plusieurs ardillons. Elle se termine par le dard.

\section{Les prises de mesures classiques}

Deux articles établissent la façon de mesurer un hameçon en l'orientant verticalement selon un axe perpendiculaire à la courbure (Garanger, 1965 : 128 ; Sinoto, 1975 : 8). Il s'inscrit ainsi à l'intérieur de deux axes de mesures, $x$ et $y$, la hampe étant placée parallèlement à l'axe vertical $(y)$ pour les hameçons à hampe droite (figure 2). Pour les hameçons à hampe droite, ce procédé donne des résultats satisfaisants. En revanche, appliquée à des hameçons à hampe courbée, la méthode montre ses limites. En effet, l'orientation de la hampe est laissée à l'appréciation de l'observateur et, selon le choix qui est fait, des mesures très variables peuvent être obtenues (figures 3 et 4). Cette méthode de prise de mesures est utilisée, par exemple, par P. Ottino (1992-a : 74), qui analyse les 139 pièces de la collection du site d'Anapua (Ua Pou, Iles Marquises) et par A. Skjølsvold (1972: 26) sur la collection du site de Hanapete'o (Hiva Oa, Iles Marquises). 


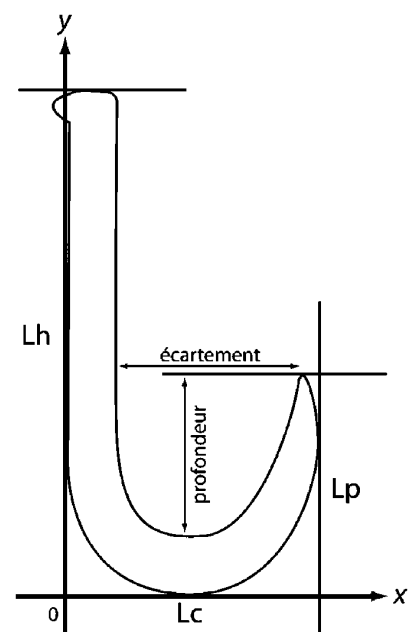

Figure 2. - Méthode classique de prise de mesures pour les hameçons à hampe droite

$(\mathrm{Lh}=$ longueur de la hampe, $\mathrm{Lc}=$ longueur de la courbure, $\mathrm{Lp}=$ longueur de la pointe.)

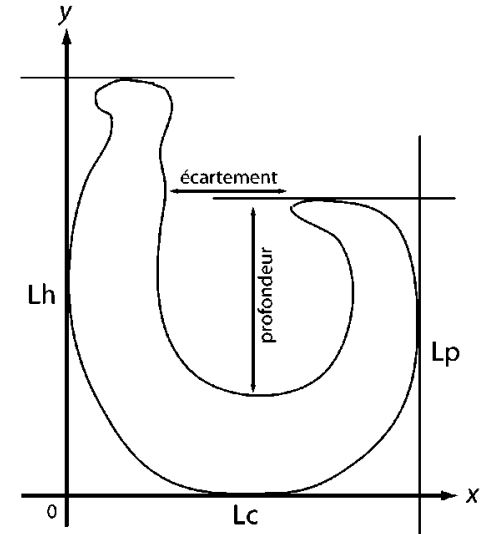

Figure 3. - Méthode classique de prise de mesures pour les hameçons à hampe courbée
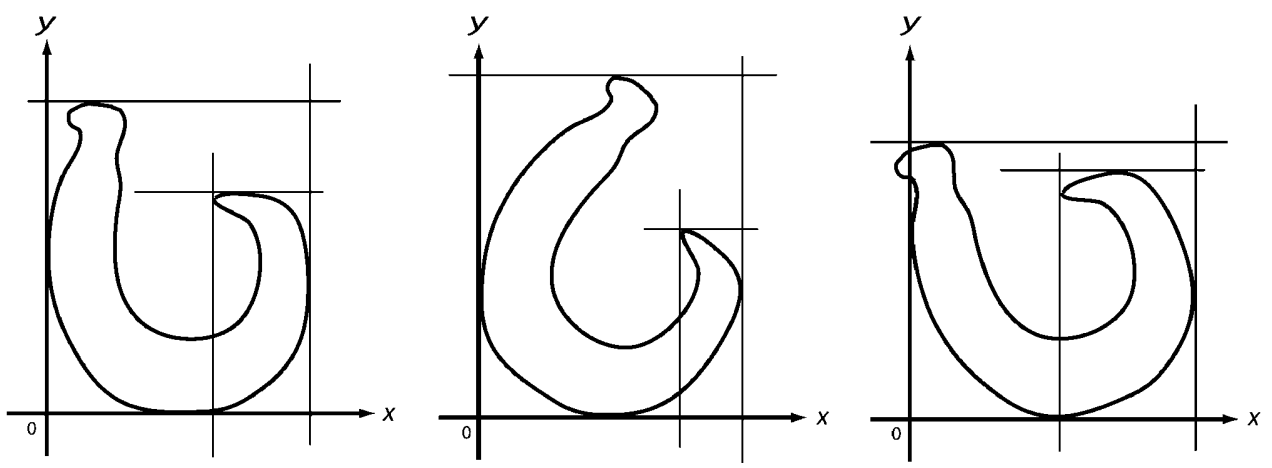

Figure 4. - Démonstration des marges d'erreurs de la méthode classique

Proposition d'une nouvelle méthode de prise de mesures

La méthode de prise de mesures que nous proposons ci-dessous se veut plus rationnelle. Elle pourra sembler complexe de prime abord, mais sa mise en ouvre est bien plus simple qu'il n'y paraît. Elle doit pouvoir s'appliquer à toutes les formes d'hameçons sans que les mesures prises soient susceptibles de varier selon leur auteur. La procédure peut se décomposer en quatre étapes :

- Étape 1 : L'hameçon est cadré sur deux axes $x$ et $y$, l'angle intérieur de la tête étant disposé sur le point 0 et la pointe mise en contact avec l'axe $x$ (figure 5).

- Étape 2 : On trace la tangente extérieure à la courbure, puis une droite à partir du point 0 et perpendiculaire à la tangente. Elle donne ainsi une première mesure : la longueur de la hampe qui est aussi celle de l'hameçon (figure 6a).

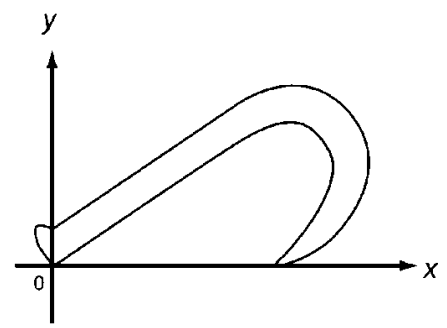

Figure 5. - Schéma de la nouvelle méthode de mesure : étape 1

- Étape 3 : Un deuxième axe est tracé au-dessus de la tête, parallèlement à la tangente et donc perpendiculairement à l'axe indiquant la longueur de l'hameçon (figure 6b).

- Étape 4 : L'hameçon est inclus dans un cadre comprenant les deux lignes déjà tracées et celles qui leur sont perpendiculaires et qui entrent en contact avec l'extrémité du dard et la partie inférieure de la hampe (figure 6c). 

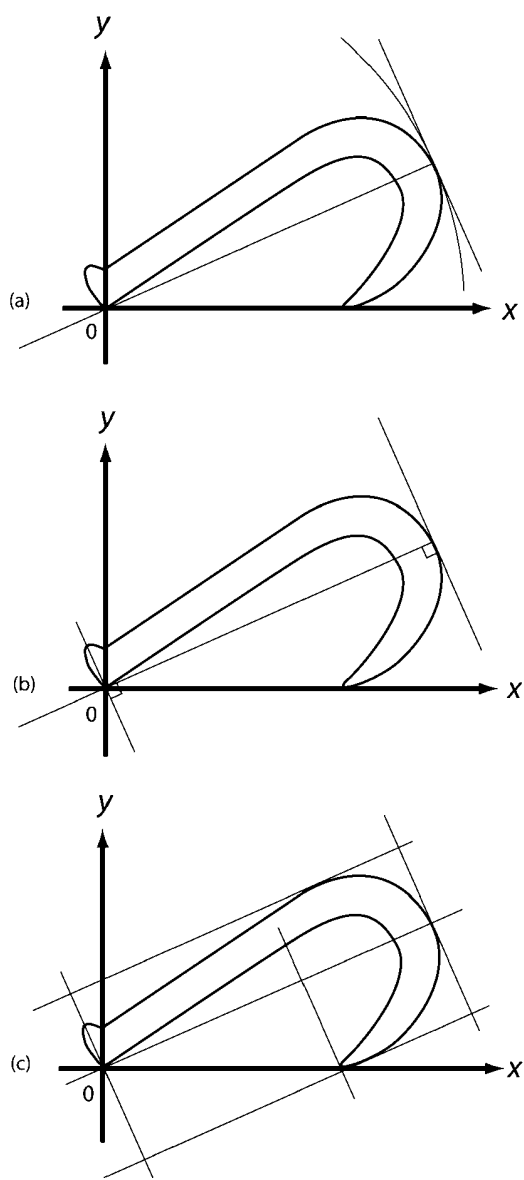

Figure 6. - Schéma de la nouvelle méthode de mesure : étapes 2,3 et 4

Ce cadre fixe sert de base à la prise de toutes les mesures utiles pour l'étude des hameçons, quelle que soit la forme de sa hampe, de sa courbure ou de sa pointe. L'objet ayant été disposé à partir de deux points constants et invariables présents sur tous les hameçons (la tête et le dard), n'importe qui utilisant la même procédure obtiendra des valeurs identiques pour toutes les mesures prises, ce qui n'était pas le cas avec la méthode employée à l'ordinaire. La figure 7 indique comment les mesures prises d'ordinaire sur les hameçons peuvent l'être une fois l'hameçon placé de la façon préconisée ici. La longueur de la hampe est aussi celle de l'hameçon; la longueur de la courbure est la largeur de l'hameçon, la longueur de la pointe, l'écartement de la hampe à la pointe ainsi que la profondeur de l'hameçon.

\section{Méthode de reconstitution des hameçons initiaux à partir des hameçons partiels et des fragments}

Les collections d'hameçons, sont, pour la plupart, surtout constituées de fragments. D'ordi-

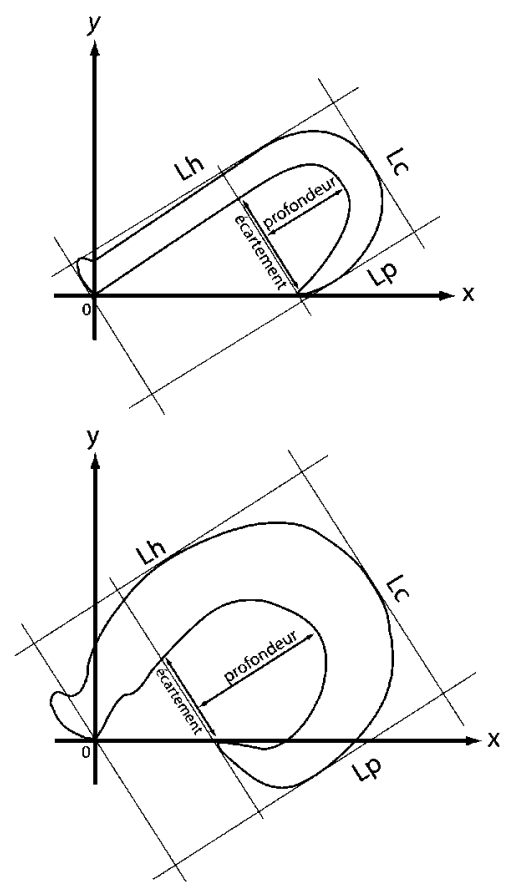

FIgURE 7. - Mesures prises sur les hameçons positionnés selon la nouvelle méthode

naire, ces vestiges sont peu étudiés et l'on se contente le plus souvent d'un comptage en fonction de la partie (ou des parties) de l'hameçon qu'ils représentent. Nous avons souhaité aller au-delà et, pour la première fois à notre connaissance, essayé, à partir des fragments en notre possession, de déterminer le type et la taille initiale des hameçons entiers dont ils proviennent. Cette reconstitution des hameçons entiers à partir des fragments permettra de posséder une connaissance bien plus étendue du matériel de pêche dont disposaient les habitants des sites d'où proviennent les hameçons découverts, ce qui autorisera une restitution plus fine de leurs méthodes de pêche.

Le principe de base de cette démarche est de prendre comme référence les hameçons entiers ou partiels bien conservés retrouvés en fouille. En effet, pour peu qu'ils soient en nombre suffisant, nous pensons qu'ils sont représentatifs de l'éventail des types et des tailles employés par les anciens habitants du site étudié et que quasiment tous les fragments retrouvés doivent avoir appartenu à des hameçons proches de ceux dont nous possédons des exemplaires bien conservés.

La méthode que nous proposons, que nous avons nommée " méthode graphique », est d'une grande simplicité de mise en œuvre. Il s'agit de confronter le fragment aux hameçons entiers 


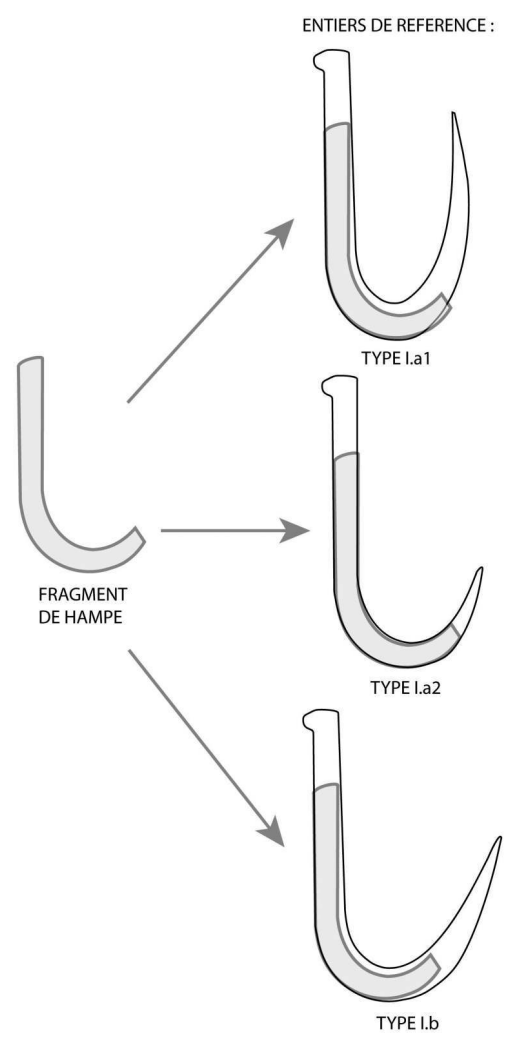

FIGURE 8. - Reconstitution d'un hameçon simple par la méthode graphique

présents dans la collection afin d'en déduire non seulement son type d'origine mais aussi, avec plus ou moins de précision, les dimensions de l'hameçon initial. Cette confrontation se fait en superposant le fragment à des copies d'hameçons entiers, obtenues par photocopie ou scanner (figure 8). Pour aider à l'identification des types et surtout à la détermination des tailles des hameçons initiaux d'où proviennent les fragments, les hameçons de référence sont reproduits selon différents modules permettant de disposer d'un éventail de dimensions plus large.

Nous allons tester la pertinence de cette méthode en l'appliquant à l'analyse d'un corpus provenant du site dunaire de Manihina (île de Ua Huka, Marquises) qui présente l'avantage de comporter 646 pièces (hameçons, fragments, ébauches, etc.) associées à l'exploitation des ressources marines, ce qui permet d'obtenir des résultats statistiquement significatifs.

\section{Reconstitution des hameçons du site de Manihina (Ua Huka, îles Marquises)}

Ua Huka est l'une des quatre îles du groupe Nord de l'archipel des Marquises, le plus septentrional de la Polynésie française. Le site de Manihina, au débouché de la vallée du même nom, se trouve sur la côte sud de l'île (figure 9). Découvert au début des années 1960, cinq sondages y ont été effectués par Y. H. Sinoto et M. Kellum sur les zones basses, côté mer (Sinoto et Kellum, 1965 : 29, 30). En 1991 et 1993, É. Conte organisa trois campagnes de fouilles, cette fois sur le sommet de la dune, qui, entre autres, ont donné un riche matériel lié à l'exploitation des ressources marines (Conte, 2002), notamment des hameçons en nacre entiers ou fragmentés, des ébauches d'hameçons, des déchets du travail de la nacre, etc., pour un total de 646 pièces.

Sur les 646 pièces découvertes liées au matériel de pêche, 493 sont des hameçons et des fragments d'hameçons finis, 90 sont des hameçons ou des fragments d'hameçons inachevés et 61 sont des morceaux de nacre travaillée très probablement pour la fabrication d'hameçons. Il faut noter la présence de deux pièces dont il est difficile de déterminer si elles sont finies ou non (tableau 1).

\begin{tabular}{|c|c|c|}
\hline & Hameçons finis & $\begin{array}{c}\text { Pièces } \\
\text { inachevées }\end{array}$ \\
\hline Hameçons simples, dont & 480 & \\
\hline - hameçons entiers & 23 & \\
\hline - hameçons partiels & 63 & \\
\hline - fragments & 394 & \\
\hline Hameçons composés & 13 & \\
\hline Ébauches & & 90 \\
\hline Nacres travaillées & & 61 \\
\hline Total des pièces définies & 493 & 151 \\
\hline Pièces indéterminées (finies ou inachevées) & \multicolumn{2}{|c|}{2} \\
\hline TOTAL du corpus & \multicolumn{2}{|c|}{646} \\
\hline
\end{tabular}

TABlEau 1. - Effectif du corpus 


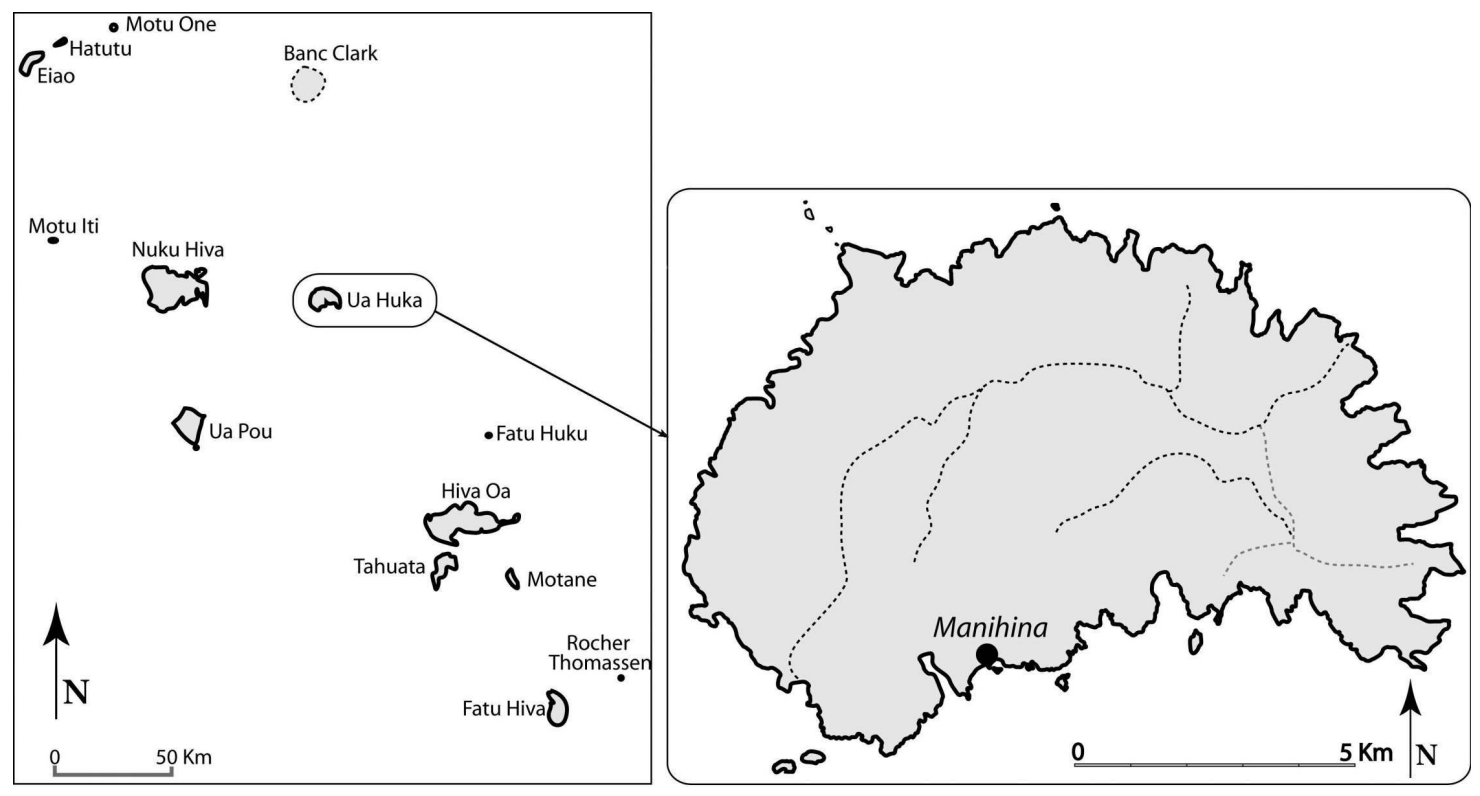

Figure 9. - Localisation du site Manihina, sur l'île de Ua Huka, archipel des Marquises

Sur les 493 pièces appartenant à des hameçons finis, 480 pièces, soit plus de $97 \%$, appartiennent à des hameçons simples, tandis que 13 seulement, soit 2,6\%, correspondent à des hameçons composés, constitués d'au moins deux éléments. Cette proportion est favorable à notre analyse dans la mesure où la méthode de reconstitution porte sur les hameçons simples. Les fragments sont au nombre de 394 sur 646 pièces, soit près de $61 \%$ de la collection, ce qui nous offre un échantillon suffisant pour tester la validité de la méthode.

\section{Les catégories de vestiges pris en compte}

Nous avons travaillé sur 63 hameçons partiels et sur un total de 394 fragments. Précisons la distinction que nous établissons entre hameçons partiels et fragments d'hameçons : les premiers comportent la tête, la hampe et suffisamment de courbure pour définir leur hauteur totale. Les fragments ne sont que l'une de ces parties (une hampe, par exemple), voire même un morceau d'une de ces parties (un fragment de hampe). Nous comptons ainsi 394 fragments divers, dont 165 fragments de hampe, 128 fragments de tête, 41 fragments de courbure et 60 fragments de pointe (tableau 2)

Comme cela a été préconisé plus haut, pour permettre la mise en œuvre de la méthode, nous avons établi, pour chaque hameçon entier de chaque type présent dans notre corpus, une représentation de tous ses modules selon une variation de la longueur de hampe par intervalle de $5 \mathrm{~mm}$. C'est sur ces modèles, qui représentent un bien plus grand échantillon de référence que la seule collection des entiers, que seront superposés les fragments qu'il s'agira d'étudier (figure 10).

\section{La détermination du type}

Il faut rappeler brièvement que, sur la base des hameçons entiers et de certaines pièces fragmentées mais reconnaissables, trois grands types morphologiques ont été distingués dans la collection provenant du site de Manihina. Le critère principal de définition des types est la forme de la hampe et des caractères secondaires (forme de la pointe, de la courbure...) permettent de définir des sous-types qui affinent la classification (figure 11).

\begin{tabular}{|l|c|}
\hline & Effectif \\
\hline Hameçons partiels & 63 \\
\hline Fragments, dont : & 394 \\
\hline - Fragments de hampe (avec ou sans tête) & 165 \\
\hline - Fragments de tête & 128 \\
\hline - Fragments de courbure & 41 \\
\hline - Fragments de pointe & 60 \\
\hline Total & 457 \\
\hline
\end{tabular}

TABLEAU 2. - Nombre d'hameçons partiels et répartition des fragments par catégories 


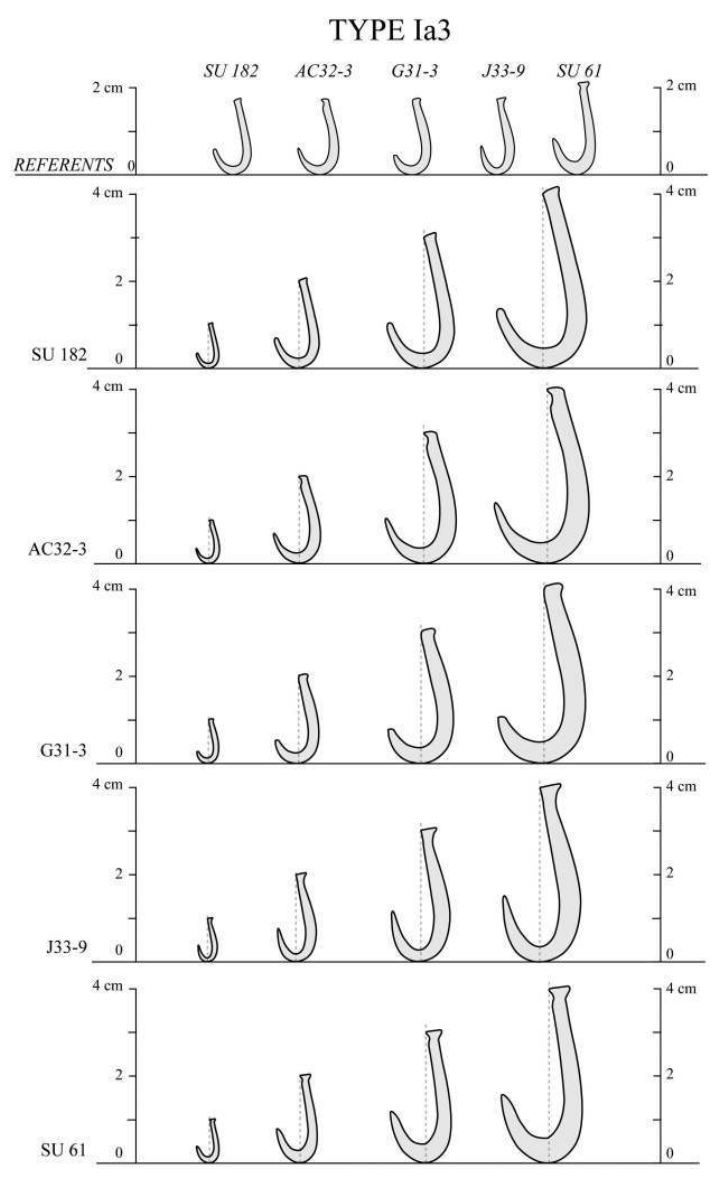

Figure 10. - Exemple des différentes catégories de taille pour les hameçons entiers de référence de type I.a (hameçons à hampe droite et pointe droite parallèle)

- Le type I se caractérise par une hampe droite (sont concernés, 75 hameçons dont 16 entiers et 59 partiels). D'après la forme de la pointe, on a défini deux sous-types :

- le sous-type I.a possède une pointe droite et parallèle à la hampe ${ }^{1}$ ( 45 hameçons, dont 12 entiers et 33 partiels appartiennent à ce sous-type). Ce type d'hameçon est divisé en trois sous-groupes selon la longueur de la pointe (41 hameçons, dont 12 entiers et 29 partiels ont ainsi été répartis car 4 partiels ont uniquement été déterminés au niveau du sous-type I.a) :

- I.a 1. Ce sont des hameçons «profonds ", dont la longueur de la pointe est supérieure à la moitié de la longueur de la hampe $(\mathrm{Lp}>1 / 2 \mathrm{Lh})(7$ hameçons dont 4 entiers et 3 partiels sont concernés) ;

- I.a 2. Ce sont des hameçons dits « égaux » caractérisés par une longueur

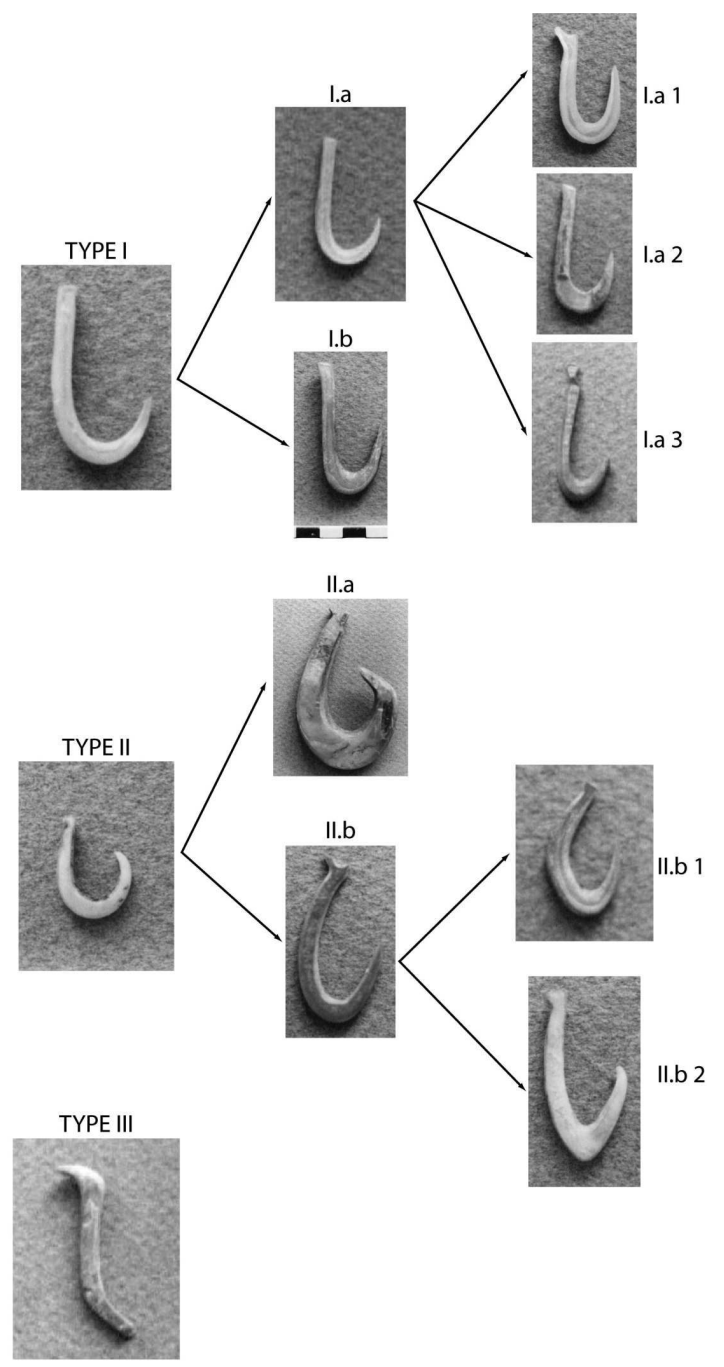

FIGURE 11. - Tableau de classification morphologique

de pointe égalant la moitié de celle de la hampe $(\mathrm{Lp}=1 / 2 \mathrm{Lh})$ (11 hameçons dont 3 entiers et 8 partiels);

- I.a 3. Ce sont des hameçons " courts" dont la longueur de la pointe est inférieure à la moitié de la longueur de la hampe $(\mathrm{Lp}<1 / 2 \mathrm{Lh})(23$ hameçons dont 5 entiers et 18 partiels);

- le sous-type I.b concerne les hameçons possédant une pointe droite et divergente par rapport à l'axe de la hampe ${ }^{2}$. Il regroupe 16 hameçons dont 4 entiers et 12 partiels.

- Le type II. Ce sont des hameçons à hampe courbée. Sont concernés 11 hameçons dont 7 entiers et 4 partiels. Nous avons subdivisé ce type en deux sous-types : 
- le sous-type II.a : c'est un hameçon dit « circulaire », avec une hampe et une pointe courbées $^{3}$ ( 2 entiers) ;

- le sous-type II.b qui possède une hampe courbée associée à une pointe droite $(9$ hameçons dont 5 entiers et 4 partiels). Deux sous-divisions sont également établies en fonction de la forme de la courbure :

- II.b 1: la courbure est arrondie 4 (7 hameçons dont 4 entiers et 3 partiels) ;

- II.b 2: la courbure est en $\langle\mathrm{V}\rangle^{5}(2$ hameçons, dont 1 entier et 1 partiel).

- Le type III se caractérise par une hampe coudée ou formant un angle ${ }^{6}$. Seulement 2 fragments appartiennent à ce type.

- Identification du type des hameçons partiels (63 au total)

Ce sont les pièces les plus faciles à identifier car il ne leur manque qu'une seule zone, à savoir au moins une partie de la pointe. La plupart de ces pièces sont associées à des hameçons de type I, c'est-à-dire à hampe droite : 33 pour le type I.a, à pointe parallèle, et 12 pour le type I.b, à pointe divergente. Quatorze hameçons partiels ont également été associés à ce type sans plus de précision au niveau du sous-type, leur pointe n'étant pas assez bien conservée pour permettre de les identifier avec certitude.

Seuls 4 exemplaires ont été reconnus comme étant des hameçons de type II, à hampe courbée, plus exactement de type II.b, à pointe droite (trois de II.b 1 - courbure en " $\mathrm{U}$ » - et un de II.b 2 - courbure en « V »).

\begin{tabular}{|l|c|}
\hline \multicolumn{1}{|c|}{ TYPES } & $\begin{array}{c}\text { Hameçons } \\
\text { partiels } \\
\text { identifiés }\end{array}$ \\
\hline Type I & $\mathbf{5 9}$ \\
\hline Déterminés seulement au niveau du type (I) & 14 \\
\hline I.a & 33 \\
\hline I.b & 12 \\
\hline Type II & $\mathbf{4}$ \\
\hline II.b & 4 \\
\hline Total identifié sur 63 & $\mathbf{6 3}$ \\
\hline
\end{tabular}

TABLEAU 3. - Pièces identifiées par type à partir des hameçons partiels

Tous les hameçons partiels ont donc pu être classés par types ( $c f$. tableau 3 ). De plus, pour 45 des 59 hameçons de type I (soit pour 76,3\%), il a été possible grâce à cette méthode de faire la distinction entre hameçon de type I.a et de type I.b.

Les fragments de hampe (avec ou sans tête et avec ou sans début de courbure) sont beaucoup plus nombreux, avec 165 pièces (tableau 4). Il est encore aisé, comme pour les fragments précédemment analysés, d'identifier leur morphologie générale car la définition de nos types s'est faite à partir de la forme des hampes. On verra que l'on peut aussi estimer facilement la taille des hameçons initiaux.

Sur 165 fragments de hampe, 137 ont été identifiés comme des hampes de type I, dont 74 de type I.a (en majorité de I.a 3, les hameçons « courts ») et 7 de type I.b. Pour 56 fragments, le sous-type n'a pas pu être déterminé avec plus de précision.

Seulement 17 ont été associés au type II, sans plus de précision, et 2 au type III. Pour seulement 9 fragments, il n'a pas été possible de définir quel était le type de l'hameçon d'origine. En définitive, le pourcentage de réussite est de plus de $94,5 \%$ pour les fragments de hampe.

\begin{tabular}{|l|c|}
\hline \multicolumn{1}{|c|}{ TYPES } & $\begin{array}{c}\text { Fragments } \\
\text { de hampes } \\
\text { identifiés }\end{array}$ \\
\hline Type I & $\mathbf{1 3 7}$ \\
\hline Déterminés seulement au niveau du type (I) & 56 \\
\hline I.a & 74 \\
\hline I.b & 7 \\
\hline Type II & $\mathbf{1 7}$ \\
\hline Déterminés seulement au niveau du type (II) & 6 \\
\hline II.a & 1 \\
\hline II.b & 10 \\
\hline Type III & $\mathbf{2}$ \\
\hline Indéterminés & $\mathbf{9}$ \\
\hline Total des hameçons identifiés sur $\mathbf{1 6 5}$ & $\mathbf{1 5 6}$ \\
\hline
\end{tabular}

TABlEAU 4. - Pièces identifiées par types à partir des fragments de hampe

Restent les fragments de courbure et de pointe qui sont les parties les plus difficiles à identifier car notre critère de classification par types repose sur la forme des hampes. Or les fragments de pointe et de courbure peuvent être semblables pour des hameçons à hampe droite et pour des hameçons à hampe courbée.

3. Ils sont appelés circular hook ou rotating hook dans la terminologie anglo-saxonne.

4. Cette forme, qui est la plus fréquente, est nommée curved shank hook par les auteurs anglo-saxons.

5. Ce sont les hameçons qui, dans la terminologie ango-saxonne, sont nommés acute ou obtuse recurved point hook.

6. Dans la terminologie anglo-saxonne, on le désigne par les expressions angular shank, bent upper shank hook, bent shank neck. 


\section{$\underline{\text { Les courbures }}$}

L'effectif total des fragments de courbures est de 41 (tableau 5). Nous avons identifié 28 courbures comme étant de type I (dont 21 de type I.a et 1 de type I.b), une courbure est associée à un hameçon de type II (II.b 1). Douze fragments de courbure n'ont pu être associés à un type d'hameçon. La méthode a donc permis d'identifier le type de $70,7 \%$ des pièces étudiées.

\begin{tabular}{|l|c|}
\hline \multicolumn{1}{|c|}{ TYPES } & $\begin{array}{c}\text { Fragments } \\
\text { de courbures } \\
\text { identifiés }\end{array}$ \\
\hline Type I & 28 \\
\hline Déterminés seulement au niveau du type (I) & 6 \\
\hline I.a & 21 \\
\hline I.b & 1 \\
\hline Type II & $\mathbf{1}$ \\
\hline II.b 1 & 1 \\
\hline Indéterminés & $\mathbf{1 2}$ \\
\hline Total identifié sur $\mathbf{4 1}$ & $\mathbf{2 9}$ \\
\hline
\end{tabular}

TABleau 5. - Pièces identifiées par types à partir des fragments de courbure

\section{Les pointes}

Parmi les 60 pointes conservées, 45 ont été identifiées comme étant des pointes d'hameçons de type I, dont 30 de type I.a et 10 de type I.b. Quatre pointes sont associées au type II, dont 3 à II.b, et aucune au type III (tableau 6). Il reste en définitive 11 pointes non identifiées. Donc, $81,6 \%$ des fragments de pointe ont pu être attribués à un type.

\begin{tabular}{|l|c|}
\hline \multicolumn{1}{|c|}{ TYPES } & $\begin{array}{c}\text { Fragments } \\
\text { de pointe } \\
\text { identifiés }\end{array}$ \\
\hline Type I & $\mathbf{4 5}$ \\
\hline Déterminés seulement au niveau du type (I) & 5 \\
\hline I.a & 30 \\
\hline I.b & 10 \\
\hline Type II & $\mathbf{4}$ \\
\hline Déterminés seulement au niveau du type (II) & 1 \\
\hline II.b & 3 \\
\hline Indéterminés & $\mathbf{1 1}$ \\
\hline Total identifié sur 60 & $\mathbf{4 9}$ \\
\hline
\end{tabular}

TABleaU 6. - Pièces identifiées par types à partir des fragments de pointe

\section{Les têtes}

Elles sont les fragments qui posent le plus de difficultés (tableau 7). Lorsqu'elles comportent un fragment de hampe, il est cependant possible de formuler une hypothèse sur le type de hameçon auquel elles appartiennent. Cependant, cette identification est moins sûre que celle réalisée sur les autres catégories de fragments. Cent vingt-huit têtes ont été étudiées et, pour 121 d'entre elles, on a pu déduire - certes avec une marge d'erreur - le type d'hameçon auquel elles appartenaient. Cent seize sont classées dans le type I, et seulement 5 comme ayant appartenu à des hameçons de type II. Malgré une certaine imprécision, il a été possible d'attribuer à des types d'hameçons 121 fragments de têtes sur 128 , soit $94,5 \%$ du corpus de têtes.

\begin{tabular}{|l|c|}
\hline \multicolumn{1}{|c|}{ TYPES } & $\begin{array}{c}\text { Fragments } \\
\text { de têtes } \\
\text { identifiés }\end{array}$ \\
\hline Type I & 116 \\
\hline Déterminés seulement au niveau du type (I) & 66 \\
\hline I.a & 50 \\
\hline Type II & 5 \\
\hline Déterminés seulement au niveau du type (II) & 2 \\
\hline II.b & 3 \\
\hline Indéterminés & 7 \\
\hline Total identifié sur $\mathbf{1 2 8}$ & 121 \\
\hline
\end{tabular}

TABLEAU 7. - Pièces identifiées par types à partir des fragments de têtes

Au final, cette méthode, malgré quelques difficultés d'interprétation pour les pièces les plus fragmentaires, a permis l'identification de nombreux morceaux d'hameçons auparavant sans intérêt réel pour les analyses typologiques. Sur les 457 pièces analysées, hameçons partiels et fragments, il a été possible de déterminer le type d'origine pour 418 d'entre elles, soit un taux de réussite de $91,4 \%$ (tableau 8 ). $\mathrm{Si}$ on se limite aux fragments, sur les 394 que comporte le corpus, nous avons pu définir le type pour 355 , soit $90,1 \%$, ce qui reste un résultat très satisfaisant.

Sur les 418 pièces identifiées, nous en comptons 385 associées au type I, ce qui donne, avec l'ajout des 16 hameçons entiers appartenant à ce type, un résultat final de 401 pièces de type I. Le type II compte 31 hameçons partiels et fragments qui, avec les 7 entiers de ce type, font un total de 38 pièces de type II. Le type III ne compte que deux exemplaires identifiés par deux fragments de hampe caractéristiques (tableau 9). 


\begin{tabular}{|c|c|c|c|c|c|c|c|}
\hline \multirow{2}{*}{$\begin{array}{l}\text { Types de } \\
\text { pièces }\end{array}$} & \multicolumn{3}{|c|}{$\begin{array}{c}\text { Nombre de pièces identifiées } \\
\text { par types }\end{array}$} & \multirow{2}{*}{$\begin{array}{l}\text { Nombre de } \\
\text { fragments non } \\
\text { déterminés }\end{array}$} & \multirow{2}{*}{$\begin{array}{c}\text { Total des } \\
\text { pièces } \\
\text { analysées }\end{array}$} & \multirow{2}{*}{$\begin{array}{c}\text { Total des } \\
\text { pièces } \\
\text { déterminées }\end{array}$} & \multirow{2}{*}{$\begin{array}{c}\text { Taux de } \\
\text { réussite } \\
\text { en } \%\end{array}$} \\
\hline & Type I & Type II & Type III & & & & \\
\hline $\begin{array}{l}\text { Hameçons } \\
\text { partiels }\end{array}$ & 59 & 4 & - & - & 63 & 63 & 100 \\
\hline Hampe & 137 & 17 & 2 & 9 & 165 & 156 & 94,5 \\
\hline Tête & 116 & 5 & - & 7 & 128 & 121 & 94,5 \\
\hline Courbure & 28 & 1 & - & 12 & 41 & 29 & 70,7 \\
\hline Pointe & 45 & 4 & - & 11 & 60 & 49 & 81,6 \\
\hline \multirow{2}{*}{ Total } & 385 & 31 & 2 & \multirow{2}{*}{39} & \multirow{2}{*}{457} & \multirow{2}{*}{418} & \multirow{2}{*}{91,4} \\
\hline & & 418 & & & & & \\
\hline
\end{tabular}

TABLEAU 8. - Hameçons partiels et fragments identifiés et indéterminées

\begin{tabular}{|l|c|c|}
\hline Type I & $\begin{array}{c}\text { Hameçons de référence } \\
\text { (entiers et partiels) }\end{array}$ & $\begin{array}{c}\text { Effectif final après la } \\
\text { reconstitution à partir des } \\
\text { fragments }\end{array}$ \\
\hline Seulement déterminés au niveau du type (I) & 14 & 147 \\
\hline Seulement déterminés au niveau du sous-type (Ia) & 4 & 54 \\
\hline Ia 1 & 7 & 20 \\
\hline Ia 2 & 11 & 24 \\
\hline Ia 3 & 23 & 122 \\
\hline Total du sous-type Ia & 45 & 220 \\
\hline Total du sous-type Ib & 16 & 34 \\
\hline Total du type I & $\mathbf{7 5}$ & $\mathbf{4 0 1}$ \\
\hline Type II & \multicolumn{2}{|c|}{} \\
\hline Seulement déterminés au niveau du type (II) & 0 & 9 \\
\hline Total du sous-type IIa & 2 & 6 \\
\hline Seulement déterminés au niveau du sous-type (IIb) & 6 & 16 \\
\hline IIb 1 & 7 & 4 \\
\hline IIb 2 & 2 & 26 \\
\hline Total du sous-type IIb & 9 & $\mathbf{3 8}$ \\
\hline Total du type II & $\mathbf{1 1}$ & $\mathbf{2}$ \\
\hline Type III & $\mathbf{2}$ & $\mathbf{4 4 1}$ \\
\hline Seulement déterminés au niveau du type (III) & $\mathbf{8 8}$ & \\
\hline Total & \multicolumn{2}{|c|}{} \\
\hline
\end{tabular}

TABLEAU 9. - Synthèse de l'identification typologique du corpus total

\section{La détermination de la taille de l'hameçon}

S'il est intéressant de pouvoir déterminer le type de l'hameçon initial à partir d'un fragment, il est également souhaitable d'être en mesure d'en estimer le module. Il serait illusoire de prétendre à une grande précision dans la restitution de la taille, mais il nous semble acceptable de réussir à intégrer les fragments dans les catégories de dimensions définies pour le corpus d'hameçons entiers.
Quatre catégories de taille ont été définies par rapport à la longueur de la hampe (Lh) qui est aussi la longueur de l'hameçon :

- hameçons de petite taille : $\mathrm{Lh} \leq 10 \mathrm{~mm}$

- hameçons de taille moyenne : $10<\mathrm{Lh}<20 \mathrm{~mm}$

- hameçons de grande taille : $20 \leq \mathrm{Lh}<30 \mathrm{~mm}$

- hameçons de très grande taille $: \mathrm{Lh} \geq 30 \mathrm{~mm}$

Dans la mesure où chaque type possède ses propres proportions, cette estimation ne peut 
être entreprise que pour les fragments dont le type aura été identifié au préalable. Ainsi, les 39 fragments dont le type n'a pas été déterminé ne pourront pas bénéficier de cette étude. Dans notre travail sur les fragments, cette reconstitution du module constitue donc une deuxième étape. Autre précision : nous possédons déjà la hauteur totale des hameçons partiels (tels que définis ci-dessus) et donc leur module. Aussi, ne sont-ils pas pris en compte dans cette étape de l'analyse.

La même "méthode graphique » que celle présentée ci-dessus pour la détermination du type de l'hameçon est utilisée pour celle de sa taille. Lors de l'analyse des fragments, ces deux opérations, dont les résultats sont présentés ici successivement, se déroulent en même temps, la superposition du fragment se faisant sur un modèle donnant une indication à la fois de la forme et de la taille.

Le type avait pu être identifié pour 355 des 394 fragments et c'est donc sur ces 355 fragments que la taille a été estimée. Au final, il a été possible de déterminer la taille de 354 d'entre eux, soit un taux de réussite de 99,7\% (tableau 10).

\begin{tabular}{|c|c|c|c|c|c|}
\hline Type I & Petit & Moyen & Grand & Très grand & Total \\
\hline Seulement déterminés au niveau du type (I) & - & 12 & 83 & 38 & 133 \\
\hline $\begin{array}{l}\text { Seulement déterminés au niveau du sous-type } \\
\text { (I.a) }\end{array}$ & - & 2 & 39 & 9 & 50 \\
\hline I.a 1 & - & 5 & 7 & 1 & 13 \\
\hline I.a 2 & - & 2 & 10 & 1 & 13 \\
\hline I.a 3 & - & 9 & 78 & 12 & 99 \\
\hline Total du sous-type I.a & - & 18 & 134 & 23 & 175 \\
\hline Total du sous-type I.b & 1 & 4 & 14 & - & 19 \\
\hline Total du type I & 1 & 34 & 231 & 61 & 327 \\
\hline \multicolumn{6}{|l|}{ Type II } \\
\hline Seulement déterminés au niveau du type (II) & - & - & 5 & 4 & 9 \\
\hline Total du sous-type II.a & - & - & - & 1 & 1 \\
\hline $\begin{array}{l}\text { Déterminés seulement au niveau du sous-type } \\
\text { (II.b) }\end{array}$ & - & - & 2 & 4 & 6 \\
\hline II.b 1 & - & 1 & 6 & 2 & 9 \\
\hline II.b 2 & - & - & - & 1 & 1 \\
\hline Total du sous-type II.b & - & 1 & 8 & 7 & 16 \\
\hline Total du type II & - & 1 & 13 & 12 & 26 \\
\hline \multicolumn{6}{|l|}{ Type III } \\
\hline Seulement déterminé au niveau du type (III) & - & - & - & 1 & 1 \\
\hline Total & 1 & 35 & 244 & 74 & 354 \\
\hline
\end{tabular}

TABLEAU 10. - Fragments identifiés par types et catégories de taille

Au début de notre analyse de la collection du site de Manihina, nous connaissions tout au plus le type et le module de 86 pièces, correspondant aux hameçons entiers et aux hameçons partiels, dont les caractères sont déterminables assez facilement.

Après l'étude qui vient d'être faite, ces caractéristiques sont connues pour un total de 439 pièces (tableau 11), ce qui signifie que notre information sur le matériel dont disposaient les anciens habitants du site a été multipliée par cinq. Il nous semble qu'il s'agit là d'un progrès considérable si l'on veut reconstituer les techniques de pêche ou si l'on veut établir des comparaisons avec les objets découverts sur d'autres sites des Marquises. 


\begin{tabular}{|l|c|c|c|c|c|}
\hline Type I & Petit & Moyen & Grand & Très grand & Total \\
\hline Déterminés seulement au niveau du type (I) & - & 13 & 96 & 38 & 147 \\
\hline I.a & 1 & 41 & 154 & 24 & 220 \\
\hline I.b & 2 & 10 & 22 & - & 34 \\
\hline Total du type I & 3 & 64 & 272 & 62 & 401 \\
\hline Type II & \multicolumn{5}{|l|}{} \\
\hline Déterminés seulement au niveau du type (II) & - & - & 5 & 4 & 9 \\
\hline II.a & - & 1 & - & 2 & 3 \\
\hline II.b & - & 6 & 12 & 7 & 25 \\
\hline Total du type II & - & 7 & 17 & 13 & 37 \\
\hline Type III & \multicolumn{5}{|l|}{} \\
\hline Déterminés seulement au niveau du type (III) & - & - & - & 1 & 1 \\
\hline Total du type III & \multicolumn{7}{|l|}{} & $\mathbf{7 1}$ & $\mathbf{2 8 9}$ & $\mathbf{7 6}$ & $\mathbf{4 3 9}$ \\
\hline Total & $\mathbf{3}$ & $\mathbf{7 1}$ &
\end{tabular}

TABLEAU 11. - Synthèse du corpus total par types et catégories de taille après reconstitution

\section{Conclusion}

La nouvelle méthode de prise de mesures proposée dans cet article présente l'avantage de positionner l'hameçon à mesurer en fonction de points fixes, la tête et le dard, que tous les hameçons possèdent et donc de donner des résultats identiques quel que soit l'auteur des mesures, autorisant ainsi des comparaisons entre des données produites par des chercheurs différents à partir de plusieurs corpus. La démarche qui consiste, à l'aide de la " méthode graphique ", à tenter de tirer parti des fragments pour reconstituer le type et la taille des hameçons dont ils proviennent ne nous semble pas vaine, autant d'un point de vue technique pour l'étude de l'exploitation des ressources marines que dans une réflexion utilisant les hameçons comme marqueurs chrono-culturels. De plus, l'application de cette méthode à la collection provenant du site de Manihina nous a permis de faire la démonstration de son efficacité. Il nous semble cependant envisageable qu'un logiciel informatique puisse être élaboré pour positionner les hameçons préalablement scannés et effectuer les mesures de manière plus rapide et, aussi, grâce à une procédure de reconnaissance des formes, pour appliquer la "méthode graphique » avec davantage de célérité.

\section{BIBLIOGRAPHIE}

Allen Melinda S., 1996. Style and function in East Polynesian fish-hooks, Antiquity 70, pp. 96-116.
CARLIER Christelle, 2002. Étude typologique de la collection d'hameçons du site de Manihina (Ua Huka, îles Marquises), mémoire de Maîtrise, Université de Paris1 Panthéon-Sorbonne.

CONTE Éric, 2000, L'archéologie en Polynésie française. Esquisse d'un bilan critique, Tahiti, Au Vent des îles, $302 \mathrm{p}$.

—, 2002. Current research on the island of Ua Huka, Marquesas archipelago, French Polynesia, Asian Perspective 41 (2), pp. 259-268.

DAVIDSON Janet, 1971. Archaeology on Nukuoro atoll. A Polynesian outlier in the Eastern Caroline Is., Bulletin of the Auckland Institute and Museum 9.

_, 1976. An archeaological assemblage of simple fishhooks from Nukuoro Atoll, Journal of the Polynesian Society 76 (2), pp. 177-196.

—, 1996. Fishing on Nukuoro Atoll, in Hommage à José Garanger, Mémoire de pierre, mémoire d'homme. Tradition et archéologie en Océanie, Paris, Publications de la Sorbonne 23, coll. Homme et Société, pp. 183-201.

EmOry Kenneth P., William Bonk and Yosihiko H. Sinoto, 1959. Fishhooks, Honolulu, Hawaiian Archaeology, Bernice P. Bishop Museum Special Publication 47.

GARANGER José, 1965. Hameçons océaniens, éléments de typologie, Journal de la Société des Océanistes 21, pp. $128-136$.

Otтіno Pierre, 1992a. Anapua: abri sous-roche de pêcheurs. Étude des hameçons ( ${ }^{\text {ère }}$ partie), Journal de la Société des Océanistes 94, pp. 57-79.

_, 1992b. Anapua: abri sous-roche de pêcheurs. Étude des hameçons ( $2^{\mathrm{e}}$ partie), Journal de la Société des Océanistes 95, pp. 201-226.

Rolett Barry V., 1998. Hanamiai. Prehistoric Colonization and Cultural Change in the Marquesas Islands (East Polynesia), New Haven, Yale University, 
Department of Anthropology and The Peabody Museum, Yale University Publications in Anthropology 81, $277 \mathrm{p}$.

SkJølsvold Arne, 1972. Excavations of a habitation cave, Hanapete'o (Hiva Oa, Marquesas islands), Honolulu, Dept. of Anthropology, Bernice P. Bishop Museum Press, Pacific Anthropological Records 16, $51 \mathrm{p}$.

Sinoto Yosihiko H., 1975. Hawaiian fishhook classification and coding systems (revised 1975), Honolulu,
Dept. of Anthropology, Bernice P. Bishop Museum Press.

Sinoto Yosihiko H. and M. Kellum, 1965. Preliminary report on excavations in the Marquesas islands, French Polynesia, Honolulu.

SugGS Robert C., 1961. The archaeology of Nuku Hiva, Marquesas islands (French Polynesia), New York, Anthropological Papers of the American Museum of Natural History, 205 p., 13 pl. 


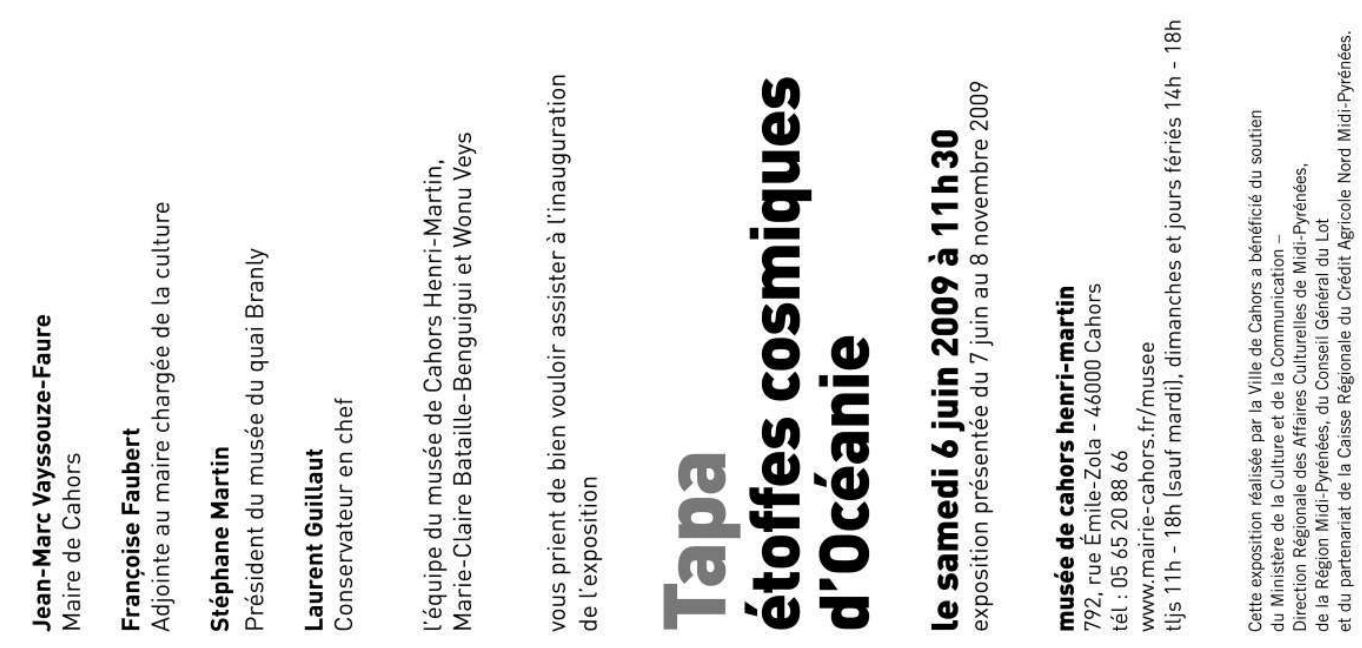

祭

圈

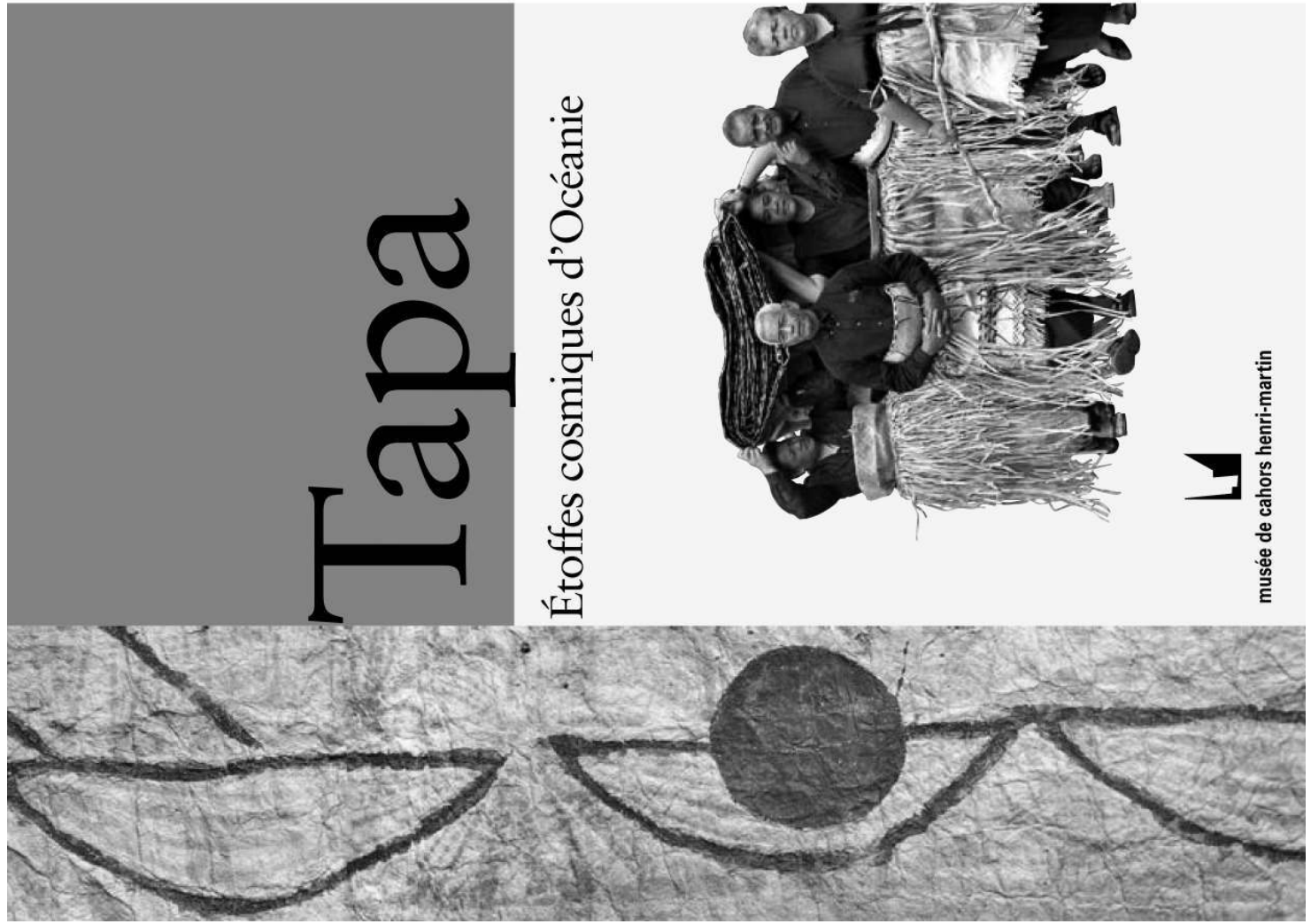

